\title{
MORBILIDAD MATERNA EXTREMA EN LA CLÍNICA UNIVERSITARIA COLOMBIA, BOGOTÁ (COLOMBIA), 2012-2013
}

\section{Extreme maternal morbidity at Clínica Universitaria Colombia. Bogotá (Colombia),} 2012-2013

Carolina del Pilar Villalba-Toquica, $M D^{\prime}$; Pablo Andrés Martínez-Silva, MD $^{2}$

Recibido: febrero 7/14 - Aceptado: diciembre 15/14

\section{RESUMEN}

Objetivo: describir la morbilidad materna extrema en una clínica de alta complejidad obstétrica.

Materiales y métodos: estudio descriptivo de vigilancia epidemiológica, basado en el análisis de los resultados de la implementación del subsistema de vigilancia de la morbilidad materna extrema (MME) en la Clínica Universitaria Colombia, entre junio de 2012 y junio de 2013. Esta es una institución de referencia que atiende pacientes de una empresa de aseguramiento privado y contributivo en Bogotá. Se estableció el número de gestantes que presentó morbilidad materna extrema en dicho periodo. Se evaluó la edad materna, la situación de salud generadora del reporte, los criterios definitorios de MME y los indicadores de calidad de la MME. Se realiza análisis descriptivo de los datos y se presenta la razón de prevalencia de la morbilidad materna extrema.

Resultados: la prevalencia de MME en la institución fue de 11 por 1.000 nacidos vivos. El 55\% de los eventos se relacionaron con hemorragias del

1 Jefe Nacional de Salud Pública, Clínicas Colsanitas. Investigadora Grupo de Investigación Gestión en Salud, Bogotá (Colombia). epifusa@gmail.com

2 Director Grupo de Investigación Gestión en Salud, Facultad de Medicina, Fundación Universitaria Sanitas, Bogotá (Colombia). embarazo, seguidos por trastornos hipertensivos del embarazo (30\%), complicaciones de comorbilidades cardiacas (9\%) y choque séptico (6\%). Un $58 \%$ de las pacientes presentaron disfunción orgánica, con una utilización de la Unidades de Cuidado Intensivo en $52 \%$; la transfusión de hemoderivados se requirió en un $61 \%$ de los eventos y la cirugía adicional en un $36 \%$. El índice de mortalidad fue del $8 \%$. La relación MME/MM fue de 11:1. El porcentaje de casos de MME con más de tres criterios fue del $73 \%$.

Conclusión: la morbilidad materna extrema es un problema frecuente en esta institución de referencia. Su medición es posible con base en la vigilancia pasiva y activa de los criterios definitorios. Se requiere más información de estos indicadores de calidad a nivel nacional para hacer comparaciones entre instituciones

Palabras clave: embarazo, mortalidad materna, morbilidad materna.

\section{ABSTRACT}

Objective: To describe extreme maternal morbidity (EMM) in a high-complexity obstetrical clinic.

Materials and methods: Descriptive epidemiological surveillance study based on the analysis of the results of the implementation of the EMM 
surveillance sub-system between June 2012 and June 2013 at Clínica Universitaria Colombia, a referral centre serving patients covered by a private health insurance company in Bogotá (Colombia). The number of pregnant women presenting with extreme maternal morbidity during that time period was determined. Maternal age, the health condition leading to the report, the EMM criteria and the EMM quality indicators were evaluated. A descriptive analysis of the data was performed. The prevalence ratio of extreme maternal morbidity is presented.

Results: The prevalence of extreme maternal mortality at the institution was found to be 11 for every 1,000 live births. Of the total number of events, 55\% were related to pregnancy bleeds, followed by gestational hypertensive disorders (30\%), complications of cardiac comorbidities (9\%), and septic shock (6\%). Of the $58 \%$ of patients who developed organ dysfunction, 52\% required admission to the Intensive Care Unit; blood product transfusion was required in $61 \%$, and additional surgery was required in 36\%. The mortality ratio was $8 \%$. The EMM/MM ratio was 11:1. The percentage of EMM cases with more than three criteria was $73 \%$.

Conclusion: Extreme maternal morbidity is a frequent problem in this referral centre. Measurement is possible on the basis of passive and active surveillance of defining criteria. Additional information about these quality indicators at a national level is required for inter-institutional comparison.

Key words: Pregnancy, maternal mortality, maternal morbidity.

\section{INTRODUCCIÓN}

La mortalidad materna (MM) sigue siendo un problema prioritario de salud pública en Colombia, a pesar de que la razón de mortalidad materna ha disminuido de 104,9 por 100.000 nacidos vivos en el año 2000 al 62,7 por $100.000 \mathrm{RN}$ vivos para el año 2008 (1); sin embargo, es mayor que la meta del país, en consonancia con los Objetivos de Desarrollo del Milenio, que es de 45 muertes maternas por 100.000 nacidos vivos (2). Acorde con la Organización Mundial de la Salud (OMS), la mayoría las muertes maternas se agrupan en torno al trabajo de parto, parto y puerperio inmediato, siendo cinco condiciones: hemorragia posparto, sepsis puerperal, preeclampsia y eclampsia, parto obstruido o prolongado, y complicaciones del aborto inseguro, las que contribuyen con al menos el $60 \%$ de todos los casos, y se consideran evitables en un 88 a $98 \%$ con el acceso oportuno a las intervenciones obstétricas pertinentes (3).

Estrechamente ligada a la mortalidad materna se encuentra la morbilidad materna extrema (MME), que se define como el caso de una mujer que estuvo a punto de fallecer, pero sobrevivió a una complicación ocurrida durante el embarazo, el parto o los 42 días siguientes a este $(4,5)$. Este es un indicador de la calidad del cuidado obstétrico, ya que está dirigido a detectar las complicaciones que afectan a la gestante y que ponen en riesgo su vida. Se ha descrito que por cada muerte materna, al menos 118 mujeres sufren MME (6). Para su detección la Federación Latinoamericana de Sociedades de Obstetricia y Ginecología (FLASOG) propuso en el 2006 los criterios de inclusión para la MME (7).

La evaluación de la MM en países en vías de desarrollo se ha adelantado mediante la metodología de "tres demoras" propuesta por Maine et al. (8). Se ha informado que las demoras tipo I y II, relacionadas con la decisión de acudir a la institución por parte de las usuarias, y las barreras para el acceso a los servicios tienen aún mucha relevancia. Pero, por otra parte, siguen teniendo gran importancia los retrasos en la atención en los servicios de salud -demora tipo III-, donde los factores humanos adquieren gran relevancia en los desenlaces, y se asocian con falta de entrenamiento en la atención de las complicaciones que amenazan la vida y problemas como la escasez de personal y su motivación, tales como: número de horas de trabajo, baja remuneración, pocos incentivos laborales, 
escasa educación continuada y dificultad de acceso a insumos básicos para la atención de las gestantes (9). Este modelo ha sido utilizado también para el análisis de la MME (10).

En Colombia se ha iniciado vigilancia de la MME en hospitales y clínicas "centinelas"; sin embargo, se encuentra que muchos de los indicadores de calidad propuestos por el Ministerio de Salud y Protección Social son aún materia pendiente, lo que revela que existen aspectos por trabajar $(11,12)$, lo cual se ha documentado en países con condiciones semejantes (13).

Con el ánimo de aportar información sobre la magnitud del problema de la MME en nuestro medio, el presente estudio presenta los resultados del seguimiento a la MME en el periodo comprendido entre junio de 2012 y junio de 2013 en la Clínica Universitaria Colombia.

\section{MATERIALES Y MÉTODOS}

Estudio descriptivo de vigilancia epidemiológica, basado en el análisis de los resultados de la implementación del subsistema de vigilancia de la MME en la Clínica Universitaria Colombia durante el periodo comprendido entre junio 15 de 2012 a junio 14 de 2013. Dicha institución, de carácter privado, se encuentra ubicada al occidente de Bogotá D.C., capital de Colombia, y oferta servicios de alto nivel de complejidad para población afiliada mediante el régimen contributivo al Sistema General de Seguridad Social en Salud, así como a inscritos en un plan complementario de medicina prepagada.

Se hizo un muestreo consecutivo de la totalidad de casos de MME, que cumplieron con la definición del Sistema de Vigilancia en Salud Pública desarrollado por el Instituto Nacional de Salud de Colombia (4). Estos fueron objeto de discusión por parte del equipo de la Junta Materno-Fetal de la institución, la cual se encuentra conformada por especialistas en ginecoobstetricia, alto riesgo materno fetal, auditoría médica, epidemiología, salud pública y salud mental. El análisis de los casos se realizó siguiendo el modelo de las "tres demoras" (8), agrupando las diferentes situaciones presentadas en cada una de estas. Asimismo, se evaluó el manejo y desenlace de cada uno de los casos. Los resultados de esta Junta, recolectados mediante actas.

Se midieron las siguientes variables: la edad materna, la situación de salud generadora del reporte, los criterios definitorios de MME, la proporción por grupos de edad con más de tres criterios de inclusión, y el análisis de la encuesta a la sobreviviente, con énfasis en los eventos relacionados con control de fertilidad previo y los indicadores de calidad de la MME.

Los resultados fueron sistematizados en una base de datos en el programa Excel (Microsoft Excel 365), el cual fue procesado mediante el software Stata 12 (StataCorp LP). En este se realizaron los análisis descriptivos de frecuencia acordes con el objetivo del estudio.

Consideraciones éticas. Los datos derivados del subsistema de vigilancia son de acceso público y se garantizó la confidencialidad de la información.

\section{RESULTADOS}

En el periodo de estudio se presentaron un total de 3.002 nacidos vivos y se identificaron 33 casos de MME para una razón de prevalencia de 11 por 1.000 recién nacidos vivos. De estos, 30 casos fueron reportados por el sistema de reporte basado en las unidades generadoras de datos centinela (91\%), mientras los restantes fueron producto de la búsqueda activa institucional. Los casos reportados se presentaron en mujeres entre los 14 y 45 años, con una edad promedio de 30 años. La mayor razón de morbilidad materna extrema se presentó en los grupos de mayor y menor edad (tabla 1).

Situación de salud generadora del reporte. Un total de 18 casos (55\%) correspondieron a choque hipovolémico relacionado con: atonía uterina, sangrado posparto, hemorragia por embarazo ectópico roto y ruptura uterina; de estos, la mitad resultó en histerectomía obstétrica, el otro $50 \%$ de estos reportes se resolvieron con manejo médico. Luego, se encuentran 10 casos (30\%) en los que se presen- 


\begin{tabular}{|c|c|c|c|c|c|}
\hline Grupos de edad & Casos MME & $\%$ & $\mathbf{N V}$ & MME Global (\%) & Razón MME \\
\hline Igual o menor a 14 años & 1 & 3 & 8 & 13 & 125 \\
\hline 15 a 21 años & 2 & 6 & 353 & 1 & 6 \\
\hline 22 a 35 años & 21 & 64 & 2,080 & 1 & 10 \\
\hline$>36$ años & 9 & 27 & 561 & 2 & 16 \\
\hline Total & 33 & 100 & 3,002 & 1 & 11 \\
\hline
\end{tabular}

Fuente: Subsistema de vigilancia de MME, Clínica Universitaria Colombia.

taron complicaciones relacionadas con trastornos hipertensivos del embarazo, tales como preeclampsia y síndrome de Hellp. Tres casos (9\%) fueron resultados de complicaciones debidas a morbilidades previas: una estenosis valvular, una estenosis aórtica y una disección de aneurisma aórtico. En los otros dos casos restantes, el reporte se debió a la presencia de choque séptico. En ningún caso se presentó eclampsia.

Criterios definitorios de MME. Los 33 casos de MME sumaron 101 criterios definitorios. Entre las intervenciones más utilizadas en el manejo de los casos se encuentran la necesidad de transfusión de hemoderivados, la utilización de los servicios de Unidades de Cuidado Intensivo, seguidos por la presencia de criterios de falla orgánica y cirugía adicional (tabla 2). En la presencia de tres criterios mostró mayor frecuencia el grupo de las mujeres con más de 36 años, quienes presentaron 8 de 9 casos (89\%), seguido del grupo de edad de 22 a 35 años con 15 de 21 casos (71\%) (tabla 3).
Encuesta de sobreviviente. En 9 casos (27\%) las mujeres planificaron el embarazo, frente 24 casos (73\%) que no lo hicieron. En un $100 \%$ se presentaba aceptación del mismo. En 17 casos (52\%) no se encontraban planificando y no deseaban el embarazo; 3 casos (9\%) manifestaron estar haciendo uso del condón; 2 casos (6\%) indicaron el uso de métodos hormonales sin regularidad; 1 caso (3\%) planificaba con el dispositivo intrauterino, y un porcentaje semejante, con el método del ritmo.

Indicadores de calidad de la MME. En el periodo del estudio la institución presentó una razón de MME de 11 por cada 1.000 nacidos vivos. El índice de mortalidad fue del $8 \%$. La relación MME/MM, el cual es un indicador de impacto de las intervenciones, fue de 11:1. El porcentaje de casos de MME con más de tres criterios fue del $73 \%$.

\section{DISCUSIÓN}

Para el periodo, la razón de MME correspondió a 11 casos por 1.000 nacidos vivos, ligeramente superior

Tabla 2.

Morbilidad materna extrema. Criterios globales en la Clínica Universitaria Colombia, 2012-2013

\begin{tabular}{|l|c|c|c|}
\hline & No. total & Media & DE \\
\hline No. criterios global / Gestante & 101 & 3 & 1 \\
\hline Unidades transfundidas & 140 & 4 & 6 \\
\hline Días UCI & 63 & 2 & 2 \\
\hline Falla orgánica & 33 & 1 & 1 \\
\hline Cirugía adicional & 12 & NA & NA \\
\hline
\end{tabular}

Fuente: Subsistema de vigilancia de MME, Clínica Universitaria Colombia. 


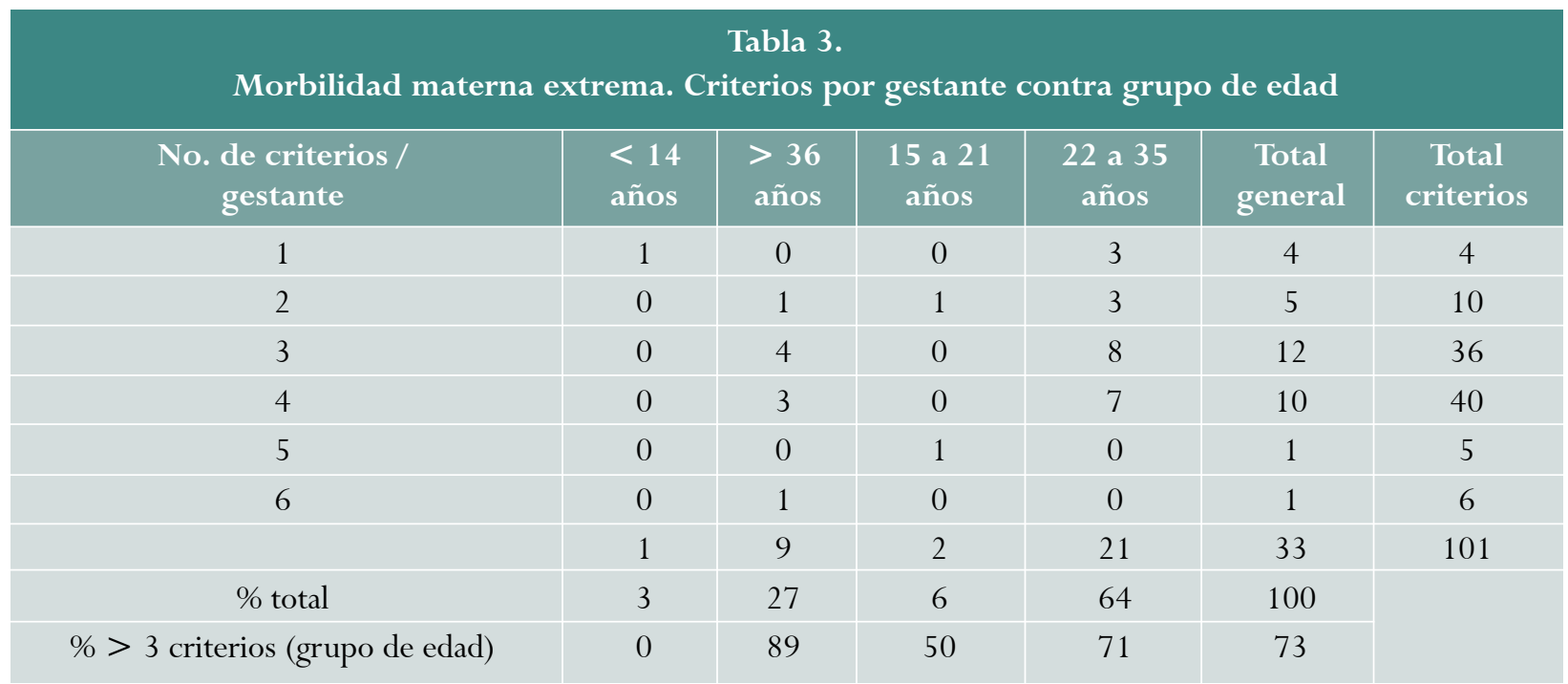

Fuente: Subsistema de vigilancia de MME, Clínica Universitaria Colombia.

a la meta nacional de $8 \times 1.000$ nacidos vivos $(4,5)$. El índice de mortalidad fue del $8 \%$, superior a la meta nacional del 4\%. La relación MME/MM fue de 11:1, menor a la meta propuesta, mayor de 35:1 (4).

Nuestros resultados son comparables a otras instituciones colombianas que han publicado sus indicadores de MME en los últimos cinco años, con una razón de prevalencia que varió entre 4 y 16 x $1.000 \mathrm{RN}$ vivos, y una relación MME/MM que varió entre 6 y 20 (11, 12). Estas tres instituciones comparten con la nuestra el papel de instituciones de referencia de nivel de complejidad semejante en atención ginecoobstétrica. Con dos de ellas se comparte el carácter universitario, y con la otra el carácter privado. Todas reciben población afiliada al régimen contributivo del Sistema General de Seguridad Social en Salud, y en dos de ellas se recibe población del régimen subsidiado (tabla 4). En relación con otros informes de la región encontramos en Perú el estudio de Reyes-Armas y Villar en una institución universitaria de referencia ubicada en Lima, que para el periodo 2002-2009 reporta una prevalencia de MME del 9,4 x 1.000 $\mathrm{RN}$ vivos, con una relación una relación $\mathrm{MME} / \mathrm{MM}$ de 30:1 (13). En México, Calvo et al. informan una prevalencia de MME de 21 x $1.000 \mathrm{RN}$ vivos y una relación MME/MM de 14 en un hospital general de referencia ubicado en el estado de Oaxaca (14).

Las mujeres en edades extremas presentaron mayores indicadores de riesgo que el rango correspondiente de los 21 a 35 años, lo cual es semejante a lo reportado en la literatura (15).

En todas las situaciones definitorias de MME la más frecuente fue el choque hipovolémico derivado de hemorragias obstétricas, las cuales desencadenaron el uso de hemoderivados, la realización de cirugías adicionales y el monitoreo en Unidad de Cuidados Intensivos. Hallazgos para otras instituciones semejantes, anteriores a estos cinco años, se encuentran referenciados en el Protocolo de Vigilancia de Morbilidad Materna Extrema desarrollado por el Instituto Nacional de Salud (4). El choque hipovolémico como resultado de distintas complicaciones, constituye poco más de la mitad de los casos de MME presentes en la institución, seguido por los trastornos hipertensivos del embarazo. Este resultado orienta a la institución a adoptar algunas acciones de mejoramiento orientadas a preparar a los equipos para el manejo de estas dos situaciones, tal como podría ser el código rojo (16).

En términos del Sistema de Vigilancia de Salud Pública, en este año de seguimiento de la MME se observa una adecuada oportunidad del reporte, lo 


\begin{tabular}{|c|c|c|c|c|c|}
\hline \multicolumn{2}{|l|}{ Indicadores } & $\begin{array}{c}\text { Meta } \\
\text { MSPS (\%) }\end{array}$ & Bogotá* & Cartagena** $^{* *}$ & B/manga \\
\hline \multirow{4}{*}{ PAMEC } & Nacidos Vivos & 3002 & 3852 & 1383 & 2,418 \\
\hline & Muertes maternas & & 3 & 3 & 1 \\
\hline & MME & 33 & 61 & 6 & 19 \\
\hline & Total criterios de inclusión & & 101 & 186 & 23 \\
\hline $\begin{array}{l}\text { Indicador de } \\
\text { resultado }\end{array}$ & Razón de MME & $8 / 1.000 \mathrm{NV}$ & 11 & 16 & 4 \\
\hline $\begin{array}{l}\text { Calidad de la } \\
\text { atención }\end{array}$ & Índice de mortalidad (IM) & $<4 \%$ & 8 & 5 & 14 \\
\hline $\begin{array}{l}\text { Calidad de la } \\
\text { atención }\end{array}$ & Relación MME/MM & $>35$ & 11 & 20 & 6 \\
\hline $\begin{array}{l}\text { Impacto de las } \\
\text { intervenciones }\end{array}$ & Relación criterio / caso & $<5$ & 3 & 3 & 4 \\
\hline $\begin{array}{l}\text { Impacto de las } \\
\text { intervenciones }\end{array}$ & $\begin{array}{l}\text { Porcentaje de casos de } \\
\text { MME con }>3 \text { criterios (\%) }\end{array}$ & $<30$ & 73 & 43 & 50 \\
\hline
\end{tabular}

cual es un indicador de calidad favorable para la institución.

\section{CONCLUSIONES}

La morbilidad materna extrema es un problema frecuente en la Clínica Colombia. Su medición es posible con base en la vigilancia pasiva y activa de los criterios definitorios. La institución presenta indicadores de calidad que no alcanzan las metas nacionales, sin embargo, al ser comparados con algunas instituciones de características parecidas, son similares. Se requiere más información de estos indicadores de calidad a nivel nacional para hacer comparaciones entre instituciones. La vigilancia de la MME es una herramienta fundamental para la gestión institucional de las acciones en salud materna, y debe ser concebida como una parte orgánica del día a día de las instituciones.

\section{AGRADECIMIENTOS}

Al equipo partícipe de la Junta Materno Fetal de la Clínica Universitaria Colombia, Organización
Sanitas Internacional. Al grupo de internos especiales de la Unidad de Salud Pública de la Clínica Universitaria Colombia.

\section{REFERENCIAS}

1. Asivamosensalud.org Bogotá D.C., Así vamos en salud; 2004 [actualizado julio de 2012] [visitado 2013 ago 18]. Disponible en: http://www.asivamosensalud.org/ inidicadores/estado-de-salud/grafica.ver/9.

2. Programa de las Naciones Unidas para el Desarrollo (PNUD). Mejorar la salud materna. ¿Cómo vamos en Colombia? Bogotá D.C. 2013 [visitado 2013 ago 18]. Disponible en: http://www.co.undp.org/content/ colombia/es/home/mdgoverview/overview/mdg5/

3. World Health Organization. Mother-Baby Package: Implementing Safe Motherhood in Countries. Geneva, Switzerland. Maternal Health and Safe Motherhood Program; 1996 [actualizado 2006] [visitado 2013 ago 20]. Disponible en: http://whqlibdoc.who.int/ hq/1994/WHO_FHE_MSM_94.11_Rev.1.pdf?ua=1

4. Instituto Nacional de Salud. Protocolo de Vigilancia en Salud Pública. Morbilidad Materna Extrema. 
Bogotá D.C., Instituto Nacional de Salud; Colombia 2006 [actualizado junio 11 de 2014] [visitado 2014 jun 16]. Disponible en: http://www.ins.gov.co:81/ lineas-de-accion/Subdireccion-Vigilancia/sivigila/ Protocolos\%20SIVIGILA/PRO\%20Morbilidad\%20 Materna\%20Extrema.pdf

5. UNFPA Colombia. Vigilancia de la Morbilidad Materna Extrema. Documento técnico y conceptual sobre la metodología de análisis de información para la auditoría de la calidad de la atención materna. Bogotá: UNFPA Colombia; 2010.

6. Pattison RC, Hall M. Near misses: a useful adjunct to maternal death enquiries. Br Med Bull. 2003;67:231-43.

7. Vigilancia de la morbilidad materna extremadamente grave (MMEG). Santa Cruz de la Sierra: Comité de mortalidad materna FLASOG; 2007.

8. Maine D, Akalin MZ, Ward VM, Kamara A. The Design and Evaluation of Maternal Mortality Programs. New York: Center for Population and Family Health, Columbia University; 1997.

9. Knight HE. ¿Why are women dying when they reach hospital on time? A systematic review of the third delay time? PLoS One. 2013;8:3-5.

10. Lori JR, Starke AE. A critical analysis of maternal morbidity and mortality in Liberia, West Africa. Midwifery. 2012;28:67-72.

11. Bello L, Vásquez D, Rojas JA, Sará P, Cogollo M, Jaramillo JJ, et al. Indicadores de morbilidad materna extrema en una clínica universitaria de tercer nivel de complejidad. Rev Cienc Biomed. 2012;9:291-9.

12. Observatorio de Salud Pública de Santander. Informe Epidemiológico de Santander. Bucaramanga, Gobernación de Santander; 2005 [actualizado 2013] [visitado 2014 mar 20]. Disponible en: http://www.observatorio.saludsantander.gov.co/ index.php/publicaciones/informe-epidemiologicode-santander/538-informe-epidemiologico-desantander-ano-viii-numero -2-2014

13. Reyes- Armas I, Villar A. Morbilidad materna extrema en el Hospital Nacional Docente Madre-Niño San Bartolomé, Lima, 2007-2009. Rev Peru Ginecol Obstet. 2012;58:273-84.

14. Calvo O, Morales V, Fabián J. Morbilidad materna extrema en el Hospital Dr. Aurelio Valdivieso, Servicios de Salud de Oaxaca. Ginecol Obstet Méx. 2010;78:660-8.

15. Oliveira FC Jr, Surita FG, Pinto E, Silva JL, Cecatti JG, Parpinelli MA, et al. Severe maternal morbidity and maternal near miss in the extremes of reproductive age: results from a national cross- sectional multicenter study. BMC Pregnancy Childbirth. 2014;20:77. doi: 10.1186/1471-2393-14-77.

16. Vélez G, Agudelo B. Guía manejo de la hemorragia obstétrica “Código Rojo". Medellín: Gobernación de Antioquia, Servicio Seccional de Salud; 2007. 